\title{
Short-term forecasting of the prevalence of clinical trachoma: utility of including delayed recovery and tests for infection
}

Fengchen Liu', Travis C. Porco 1,2,3, Abdou Amza ${ }^{4}$, Boubacar Kadri' ${ }^{4}$, Baido Nassirou ${ }^{4}$, Sheila K. West ${ }^{5}$, Robin L. Bailey ${ }^{6}$, Jeremy D. Keenan ${ }^{1,2}$ and Thomas M. Lietman ${ }^{1,2,3^{*}}$

\begin{abstract}
Background: The World Health Organization aims to control blinding trachoma by 2020. Decisions on whether to start and stop mass treatments and when to declare that control has been achieved are currently based on clinical examination data generated in population-based surveys. Thresholds are based on the district-level prevalence of trachomatous inflammation-follicular (TF) in children aged 1-9 years. Forecasts of which districts may and may not meet TF control goals by the 2020 target date could affect resource allocation in the next few years.

Methods: We constructed a hidden Markov model fit to the prevalence of two clinical signs of trachoma and PCR data in 24 communities from the recent PRET-Niger trial. The prevalence of TF in children in each community at 36 months was forecast given data from earlier time points. Forecasts were scored by the likelihood of the observed results. We assessed whether use of TF with additional TI and PCR data rather than just the use of TF alone improves forecasts, and separately whether incorporating a delay in TF recovery is beneficial.
\end{abstract}

Results: Including TI and PCR data did not significantly improve forecasts of TF. Forecasts of TF prevalence at 36 months by the model with the delay in TF recovery were significantly better than forecasts by the model without the delay in TF recovery $(p=0.003)$. A zero-inflated truncated normal observation model was better than a truncated normal observation model, and better than a sensitivity-specificity observation model.

Conclusion: The results in this study suggest that future studies could consider using just TF data for forecasting, and should include a delay in TF recovery.

Trial registration: Clinicaltrials.gov NCT00792922

Keywords: Trachoma, Model, Mass drug administration, Forecast, Prediction

\section{Background}

The World Health Organization (WHO), the International Trachoma Initiative (ITI), Ministries of Health, and their partners aim to control blinding trachoma by 2020 by implementing surgical campaigns, antibiotic distributions, hygiene initiatives, and environmental improvements $[1,2]$. Decisions on whether to start and stop mass treatments and when to declare that control has been achieved are

\footnotetext{
* Correspondence: tom.lietman@ucsf.edu

${ }^{1}$ F.I. Proctor Foundation, University of California San Francisco, 513 Parnassus, Medical Sciences 309A, San Francisco, CA 94143-0944, USA

${ }^{2}$ Department of Ophthalmology, University of California San Francisco, San

Francisco, CA, USA

Full list of author information is available at the end of the article
}

currently based on the clinical examination data generated in population-based surveys. Thresholds are based on the district-level prevalence of trachomatous inflammation-follicular (TF) in children aged 1-9 years. Forecasts of which districts may and may not meet TF control goals by the 2020 target date could affect resource allocation in the next few years.

Unfortunately, TF is not an ideal indicator for several reasons. TF is only indirectly associated with infection with the causative agent Chlamydia trachomatis, in part because the clinical signs of trachoma may persist for months after infection has been cleared [3-7]. Also, TF is a subjective sign with only moderate reproducibility. Other indicators such as trachomatous inflammation- 
intense (TI) and more direct evidence of chlamydial infection (PCR) may provide additional information, even in forecasting the future prevalence of TF.

Recent community-randomized trials have followed multiple communities which were treated with identical mass antibiotic programs, providing datasets to test various types of forecasts. These trials have assessed the prevalence of TF, TI and chlamydial PCR. Here, we use a hidden Markov model with recent clinical trial data of biannual assessments of 24 communities from baseline through 30 months to forecast the prevalence of TF in children in each at 36 months [8]. We assess whether use of TF with additional TI and PCR data rather than just the use of TF improves forecasts, and separately whether incorporating a delay in TF recovery is beneficial; we also compare different observation models.

\section{Methods}

\section{Data collection}

Forty-eight communities were followed as part of the Niger arm of the Partnership for the Rapid Elimination of Trachoma (PRET) study. Communities were randomized to either mass antibiotics of the entire community, or antibiotics targeted just to children 12 years and younger. The 24 communities included in this study received annual antibiotic treatment of all ages. Communities were assessed at baseline and then biannually for 3 years. All individuals were offered antibiotic treatment annually, within two weeks of the assessment: children under 6 months, those allergic to macrolides, and pregnant women were offered topical tetracycline, and all others were offered a single dose of oral azithromycin (20 mg/kg for children and $1 \mathrm{~g}$ for adults).

A random sample of 100 children $0-5$ years old were selected from each community. If a community had less than 100 0-5 year-old children, then all were offered assessment. Each participating child had their upper right tarsal conjunctiva swabbed, and processed for PCR as previously described [9]. Clinical grading of the right everted superior tarsal conjunctiva was performed using a $2.5 \times$ magnifying loupe and adequate sunlight or a torch light according to the WHO simplified grading system [10] as previously described [9].

\section{Ethics statement}

This study of de-identified data received ethical approval from the Committee on Human Research of the University of California San Francisco and was carried out in accordance with the Declaration of Helsinki. A parent or guardian of any child participant provided informed consent on their behalf. The informed consent given was oral: (a) we chose verbal consent because of the low literacy rates in the study area, (b) the IRB (10.00812) approved the use of oral consent, and (c) oral consent was documented on the registration form for each study participant prior to examination in the field.

\section{Modeling methods}

We constructed a stochastic transmission model of Chlamydia trachomatis infection over time. The model contains two components: (1) change in the number of infected individuals over time due to transmission, recovery and mass antibiotic treatment with the reported coverage levels, and (2) the observed TF, TI and PCRpositive based on the number of infected individuals. For community $j(j=1, \ldots, 24)$, we assumed a population of size $N_{j}$ at the time of treatment $k(k=1,2,3$ corresponding to baseline, 12 and 24 months). We used an SIS (susceptible-infectious-susceptible) model structure, assuming that the force of infection is proportional to the prevalence of infection in the population with proportionality constant $\beta$, and a constant per-capita recovery rate $\gamma$ [11]. Between periods of treatment, we assumed that the probability $p_{i, j}^{(k)}(t)$ that there are $i$ infections in community $j$ at time $t$ after treatment time point $k$ obeys the following equations $[12,13]$ :

$$
\begin{aligned}
\frac{d p_{0, j}^{(k)}}{d t}= & \gamma p_{1, j}^{(k)} \\
\frac{d p_{i, j}^{(k)}}{d t}= & \beta \frac{(i-1)\left(N_{j}-i+1\right)}{N_{j}} p_{i-1, j}^{(k)} \\
& +\gamma(i+1) p_{i+1, j}^{(k)}-\beta \frac{i\left(N_{j}-i\right)}{N_{j}} p_{i, j}^{(k)} \\
& -\gamma i p_{i, j}^{(k)}, \text { for } 1 \leq i \leq N_{j}-1 \frac{d p_{N_{j}, j}^{(k)}}{d t}=\beta \frac{N_{j}-1}{N_{j}} p_{N_{j}-1, j}^{(k)} \\
& -\gamma N_{j} p_{N_{j, j}}^{(k)}
\end{aligned}
$$

To model treatment, we assumed that each child aged $0-5$ years in community $j$ has probability $c_{j}^{(k)}$ of receiving treatment with the antibiotic efficacy $e_{k}$ for treatment

period $k$. We modeled each treatment according to $p_{i, j}^{(k)}$

$(t=0)=\sum_{i^{\prime}=i}^{N_{j}} p_{i^{\prime}, j}^{(k, p r e)}\left(\begin{array}{c}i^{\prime} \\ i\end{array}\right)\left(1-c_{j}^{(k)}\right)^{i}\left(c_{j}^{(k)} e_{k}\right)^{i^{\prime}-i}$, where $i$, is the number of infected children aged $0-5$ years eligible for treatment, $p_{i^{\prime}, j}^{(k, p r e)}$ is the probability of $i^{\prime}$ infected children aged $0-5$ years before treatment time point $k$, and $i$ is the number of infected children aged $0-5$ years after treatment. Let $S_{j, T F}^{(l)}, S_{j, T I}^{(l)}$ and $S_{j, P C R}^{(l)}$ be the observed TF, TI and PCR-positive at each observation time point $l(l=0,1,2,3$, 4 and 5 corresponding to baseline, $6,12,18,24$ and 
30 months, respectively) for community $j$. For a community with $i$ infections, the probabilities of the observed TF, TI and PCR-positive based on $i$ infections are given by using the observation component of the Kalman filter [14] (please see Additional file 1 for more details).

\section{Sensitivity analyses}

We assessed several ways of modeling each observation (TF, and where applicable, TI and PCR). Given a true hidden prevalence (Equation 1), each observation could be specified using epidemiologically driven, traditional sensitivity and specificity, instead of the technically driven, truncated normal distribution described in Additional file 1. With the truncated normal distribution, we have several options to handle the portion of the density at zero, including no zero-inflation, a density at zero (zero-inflation \#1) proportional to the density of the normal that would have been $<0$, or a density at zero given by a free, fitted parameter. In addition, we could include a delay in TF specifically (not TI or PCR), as the follicles associated with TF are known to take months if not longer to recede. Here, we performed specific sensitivity analyses: (a) fitting the model to the observed TF instead of the observed TF, TI and PCR by simplifying Equations 2 and 3 in Additional file 1; (b) using another zero-inflated truncated normal (zero-inflation \#2) as the posterior in Equation 2 in Additional file 1 in which the density at $0 \%$ (the first of those 101 discrete units) was assumed to be a parameter between 0 and 1 ( $\eta_{T F}$ for TF, $\eta_{T I}$ for TI, and $\eta_{P C R}$ for PCR); (c) using the truncated normal as the posterior; (d) assuming no delay in TF recovery; (e) assuming that the posterior is the convolution of two binomial distributions: the distribution of the number of tested positives $i_{\text {sens }}$ from true positives $i$ in a community with $N_{j}$ individuals because of the sensitivity of a test, and the distribution of the number of tested positives $i_{\text {spec }}$ from true negatives $N_{j}-i$ because of the specificity of the test. Table 1 shows 10 scenarios of sensitivity analyses.

\section{Statistics}

Bootstrap percentile confidence intervals for results from Niger were estimated using $R$ [15], $N=10,000$. CIs for model parameter estimates were obtained from MCMC with $16384\left(2^{14}\right)$ steps after a burn-in including $8192\left(2^{13}\right)$ steps.

\section{Results}

Clinical examination (TF and TI) and PCR data were available for 24 communities with 2212 children (aged 0 to 5 years). At the baseline census, communities had a mean of 146 children (95\% CI 137 to 155) aged 0 to 5 years. The mean antibiotic coverage of children was $92.3 \%$ at baseline, $89.0 \%$ at 12 months, and $89.8 \%$ at 24 months. At baseline, the observed TF prevalence in the 24 communities ranged from $10 \%$ to $57 \%$ with a mean prevalence of $27.6 \%$ (95\% CI $21.8 \%$ to $33.9 \%$ ). The follow-up TF prevalence was $15.2 \%$ (95\% CI $11.9 \%$ to $18.8 \%$ ) at 6 months, $19 \%$ (95\% CI $14.9 \%$ to $23.2 \%$ ) at 12 months, $19.7 \%$ (95 \% CI $14.6 \%$ to $25.2 \%$ ) at 18 months, $14.7 \%$ (95\% CI $10.5 \%$ to $19 \%$ ) at 24 months, and $12.3 \%$ (95 \% CI $8.5 \%$ to $16.8 \%$ ) at 30 months. The observed community prevalence of TF at 36 months which was to be forecasted ranged from $0 \%$ to $30 \%$ with a mean prevalence of $7.6 \%$ (95\% CI $4.9 \%$ to $11.3 \%)$. The observed community prevalence of TF, TI and PCR at each biannual visit are shown in Additional file 2 .

We forecasted distributions of TF prevalence in each of 24 communities from 10 models (Fig. 1 and Additional file 3 ). The forecast distributions of the hidden (true) prevalence for each of the 10 models (Fig. 2 and Additional file 4) could be markedly different, even if the eventual observation forecasts were similar (Fig. 1 and Additional file 3). The total loglikelihood (the sum of each

Table 1 Forecast scores

\begin{tabular}{llllll}
\hline Model ID & Log likelihood & Rank & Scenario & & \\
\cline { 5 - 6 } & & & Density function & Data & Delay in TF recovery \\
\hline 1 & -71.864 & 2 & Zero-inflation \#1 & TF, TI, PCR & Yes \\
2 & -72.207 & 4 & Zero-inflation \#1 & TF only & Yes \\
3 & -74.792 & 3 & Zero-inflation \#2 & TF, TI, PCR & Yes \\
4 & -74.576 & 7 & Tero-inflation \#2 & TF only & Yes \\
5 & -76.640 & 8 & Truncated normal & TF, TI, PCR & Yes \\
6 & -76.782 & 5 & Zero-inflation \#1 & TF only & No \\
7 & -76.173 & 6 & Zero-inflation \#1 & TI, PCR & No \\
8 & -76.174 & 10 & Binomial (sensitivity and specificity) & TF, TI, PCR & Yes \\
9 & -122.234 & 9 & Binomial (sensitivity and specificity) & TF only & Yes \\
\hline
\end{tabular}




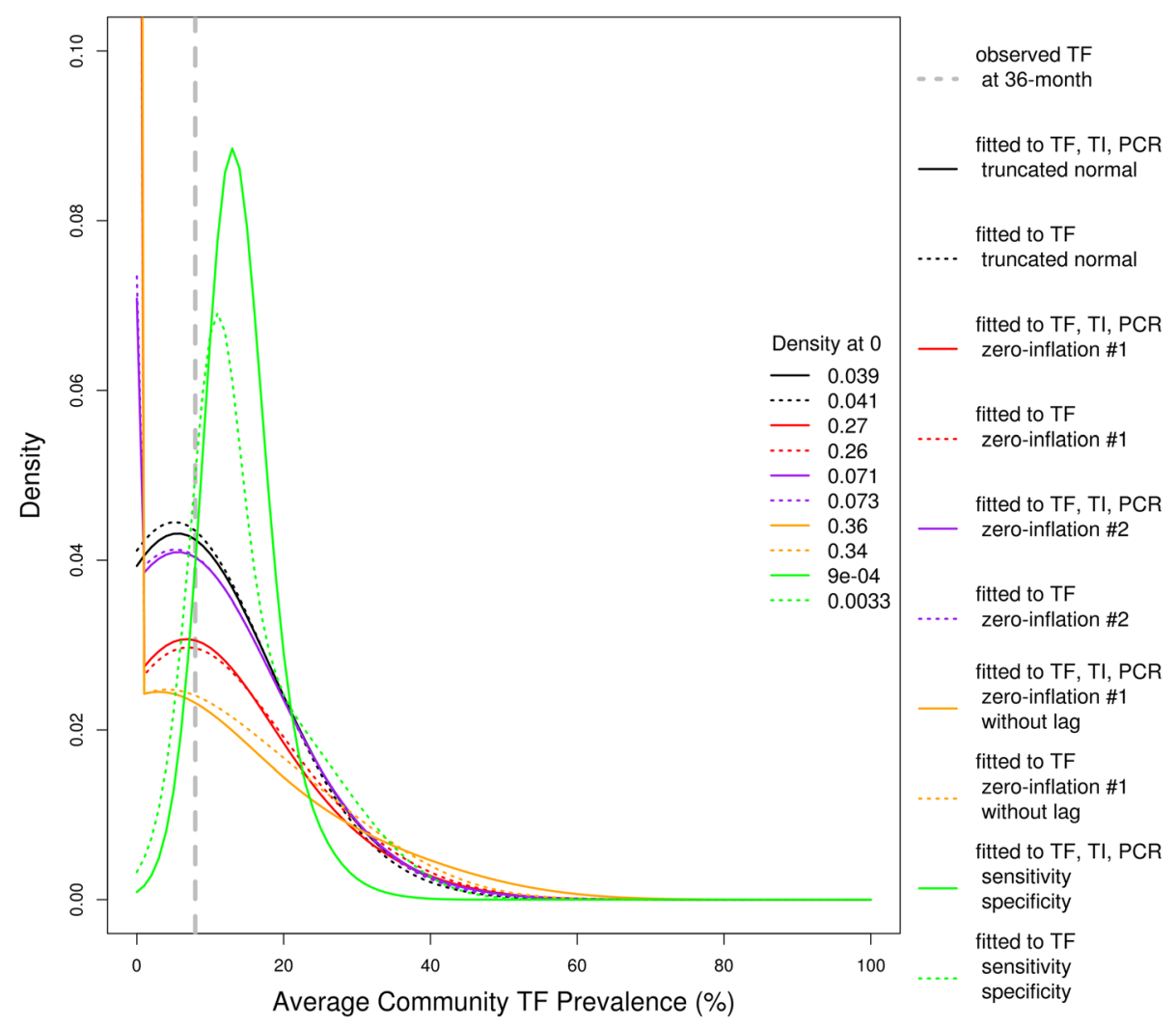

Fig. 1 Forecasts of TF prevalence versus observed TF prevalence (averaged over 24 communities). The forecast distributions of average TF prevalence at 36-month in all communities from 10 models are shown by solid and dotted curves in different colors (as listed in the large legends), and their densities at 0 are listed in the small legends. The observed average TF prevalence at 36-month is shown by the dashed grey bar. For the forecast distributions of TF and the observed TF at 36-month in each of 24 communities, please see Additional file 3

community's loglikelihood, Equation 3 in Additional file 1) of the observed 36-month TF prevalence varied between models (Table 1) given the estimated parameters (Table 2). The base case (models 1 and 2: zero-inflation \#1 with the observed TF, TI and PCR, and with the observed TF) reveals that the loglikelihood of the observed 36-month TF prevalence from the model based on the observed TF, TI and PCR data from 0 to 30 months was better than the model only based on the observed TF data from 0 to 30 months, although the difference between model 1 and model 2 was not significant (loglikelihood: -71.86 for model $1,-72.21$ for model $2, p=0.64$, Wilcoxon signed rank test).

As a sensitivity analysis, we assumed no delay in TF recovery (models 7 and 8) and compared the model without the delay in TF recovery to the base case (models 1 and 2), and found that the model with the delay in TF recovery and TF, TI and PCR data was significantly better than the model with the TF data and no delay in TF recovery (loglikelihood: -71.86 for model $1,-76.17$ for model $8, p=0.003)$, and the model with the delay in TF recovery had better likelihood than the model without the delay in TF recovery for both scenarios: multiple observations of TF, TI and PCR (loglikelihood: -71.86 for model $1,-76.17$ for model $7, p=0.002$ ), and the observation of TF only (loglikelihood: -72.21 for model 2, -76.17 for model $8, p=0.005)$. Sensitivity analyses found no significant differences between model 3 and model 4 (loglikelihood: -74.79 for model $3,-74.58$ for model $4, p=0.60$ ), model 5 and model 6 (loglikelihood: -76.64 for model 5, -76.78 for model $6, p=0.35$ ), and model 7 and model 8 (loglikelihood: -76.173 for model $5,-76.174$ for model $6, p=0.16$ ), except for model 9 and model 10 (loglikelihood: -122.23 for model 9, -105.22 for model 10, $p=0.01)$. Sensitivity analyses on models based on TF, TI and PCR data but with different posterior densities (models 1, 3, 5 and 9) show that the zero-inflation \#1 (model 1) was better than other models, and the observation model with the sensitivity and specificity did not improve forecasts and had the lowest likelihood (Table 1).

Models 9 and 10 allowed estimation of sensitivity and specificity for each of TF, TI and PCR (Table 3). TF was estimated to be $65.2 \%$ (95\% CI $61.2 \%$ to $82.3 \%$ ) sensitive and $89.7 \%$ (95\% CI $87.9 \%$ to $90.7 \%$ ) specific; the delay in TF recovery was estimated to be 0.32 per six- 


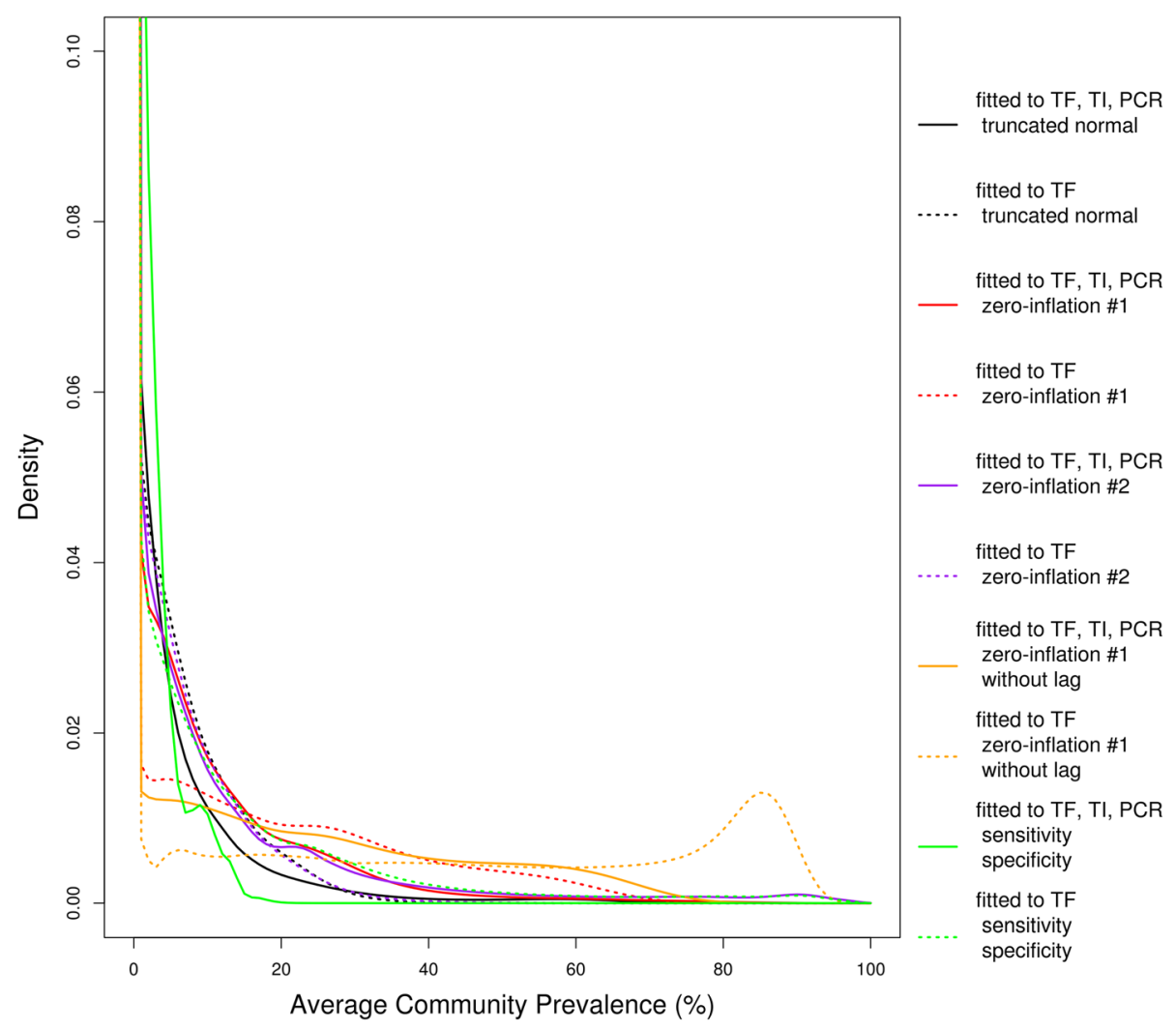

Fig. 2 Forecasts of the hidden (true) prevalence (averaged over 24 communities). The forecast distributions of average true prevalence at 36-month in all communities from 10 models are shown by solid and dotted curves in different colors (as listed in legends). For the forecast distributions of true prevalence at 36-month in each of 24 communities, please see Additional file 4

month (95\% CI 0.25 to 0.36 ); TI was estimated to be $58 \%$ (95\% CI $50.9 \%$ to $78.3 \%$ ) sensitive and $98.4 \%$ (95\% CI $98 \%$ to $98.6 \%$ ) specific, and PCR was estimated to be $62.7 \%$ (95\% CI $57.5 \%$ to $88.7 \%$ ) sensitive and $96.3 \%$ (95\% CI $95.7 \%$ to $96.6 \%$ ) specific.

\section{Discussion and conclusion}

Here, we compared the inclusion of several features of model-based forecasts of the prevalence of clinically active trachoma (TF) 6-months into the future. Inclusion of the second trachoma sign TI and lab-based chlamydia testing improved TF forecasts, but not significantly. Thus we cannot state that PCR testing is necessary for better forecasting of TF, although it may well help forecast future infection-that would require further study. $\mathrm{TF}$ is known to remain for months after infection has cleared, and inclusion of this feature into the observation model improved forecasting significantly. Different forms of the observation portion of the hidden model performed differently. In particular, a zero-inflated truncated normal distribution performed better than the observation model with sensitivity and specificity, and inclusion of the sensitivity and specificity into the observation model did not improve forecasts.
We estimated the sensitivity and specificity for each of TF, TI and PCR. Our estimates of TF sensitivity $(65.2 \%$ from $61.2 \%$ to $82.3 \%$ ) and specificity (89.7\% from $87.9 \%$ to $90.7 \%$ ) are consistent with the estimates in studies $[5,16-18]$ in Tanzania and The Gambia, in which sensitivity was estimated at $24.0 \%$ to $86.7 \%$ and specificity at $74.0 \%$ to $94.0 \%$. However, in study [3] in Ethiopia, TF sensitivity was estimated to be $87.3 \%$ (higher than our estimate of TF sensitivity), and TF specificity was estimated to be $36.6 \%$ (lower than our estimate of TF specificity). Our estimates of TI sensitivity (58.0\% from $50.9 \%$ to $78.3 \%$ ) and specificity (98.4\% from $98.0 \%$ to $98.6 \%$ ) closely agree with the estimates in $[3,5,16,17]$ in which sensitivity was estimated at $12.4 \%$ to $77.0 \%$ and specificity at $74.0 \%$ to $99.4 \%$. The estimate of PCR specificity (96.3\% from $95.7 \%$ to $96.6 \%)$ in our study is close to the estimates in $[3,5,17]$ in which PCR specificity was estimated at $93.0 \%$ to $100 \%$. However, our estimate of PCR sensitivity $(62.7 \%$ from $57.5 \%$ to $88.7 \%$ ) is lower than the estimates in $[3,5,17]$ in which PCR sensitivity was estimated at $77.8 \%$ to $97.0 \%$.

Previous mathematical models have provided insight into the transmission of trachoma [3, 5, 16, 17, 19-24], 
Table 2 Estimated parameters

\begin{tabular}{|c|c|c|c|c|c|c|c|c|}
\hline \multirow[t]{2}{*}{ Model ID } & \multicolumn{8}{|c|}{ Estimated parameters ${ }^{\mathrm{a}}$ mean $(95 \% \mathrm{Cl})^{\mathrm{b}}$} \\
\hline & Mean of $\log \beta$ & SD of $\log \beta$ & Efficacy of antibiotic & \multirow{2}{*}{$\begin{array}{l}r \\
\\
0.127(0.0561,0.191)\end{array}$} & \multirow{2}{*}{$\begin{array}{l}\mu_{T F} \\
0.816(0.634,0.995)\end{array}$} & $\sigma_{T F}$ & \multirow{2}{*}{$\begin{array}{l}\lambda_{T F} \\
0.571(0.445,0.664)\end{array}$} & $\mu_{T I}$ \\
\hline 1 & $-1.18(-2.35,-0.782)$ & $0.363(0.216,0.754)$ & $0.891(0.838,0.944)$ & & & $0.245(0.19,0.32)$ & & $0.166(0.1,0.533)$ \\
\hline 2 & $-0.57(-1.31,-0.145)$ & $0.0987(0.00192,0.341)$ & $0.888(0.839,0.952)$ & $0.231(0.057,0.47)$ & $0.657(0.294,0.983)$ & $0.231(0.178,0.303)$ & $0.55(0.438,0.644)$ & - \\
\hline 3 & $-1.68(-2.6,-1.06)$ & $0.779(0.418,0.988)$ & $0.937(0.837,0.978)$ & $0.08(0.055,0.207)$ & $0.358(0.181,0.994)$ & $0.296(0.188,0.358)$ & $0.591(0.182,0.665)$ & $0.135(0.0618,0.436)$ \\
\hline 4 & $-0.922(-7.48,-0.2)$ & $0.117(0.006,0.711)$ & $0.899(0.838,0.983)$ & $0.264(0.061,0.461)$ & $0.671(0.107,0.984)$ & $0.249(0.166,0.413)$ & $0.495(0.302,0.7)$ & - \\
\hline 5 & $-1.24(-2.52,-0.831)$ & $0.629(0.276,0.756)$ & $0.928(0.843,0.979)$ & $0.352(0.106,0.419)$ & $0.862(0.616,0.97)$ & $0.295(0.19,0.34)$ & $0.597(0.244,0.649)$ & $0.272(0.132,0.513)$ \\
\hline 6 & $-2.84(-9.56,-0.116)$ & $0.184(0.00647,0.634)$ & $0.964(0.838,0.995)$ & $0.351(0.0579,0.472)$ & $0.702(0.211,0.977)$ & $0.308(0.172,0.384)$ & $0.592(0.314,0.686)$ & - \\
\hline 7 & $-0.309(-1.31,-0.191)$ & $0.161(0.0695,0.381)$ & $0.883(0.838,0.903)$ & $0.289(0.156,0.376)$ & $0.583(0.508,0.994)$ & $0.126(0.112,0.157)$ & - & $0.0881(0.0592,0.364)$ \\
\hline 8 & $-0.123(-0.637,-0.0199)$ & $0.0411(0.001,0.164)$ & $0.859(0.837,0.896)$ & $0.0995(0.055,0.271)$ & $0.301(0.251,0.814)$ & $0.121(0.107,0.141)$ & - & - \\
\hline 9 & $-3.73(-9.74,-2.45)$ & $0.774(0.0932,0.995)$ & $0.886(0.857,0.911)$ & $0.16(0.149,0.166)$ & $0.652(0.612,0.823)$ & $0.897(0.879,0.907)$ & $0.315(0.247,0.356)$ & $0.58(0.509,0.783)$ \\
\hline 10 & $-1.53(-7.76,-1.16)$ & $0.651(0.0369,0.953)$ & $0.966(0.855,0.998)$ & $0.0833(0.0572,0.165)$ & $0.536(0.501,0.904)$ & $0.903(0.887,0.916)$ & $0.242(0.18,0.348)$ & - \\
\hline \multirow[t]{2}{*}{ Model ID } & \multicolumn{8}{|c|}{ Estimated parameters ${ }^{\mathrm{a}}$ mean $(95 \% \mathrm{Cl})^{\mathrm{b}}$} \\
\hline & \multicolumn{2}{|l|}{$\sigma_{T I}$} & $\mu_{P C R}$ & $\sigma_{P C R}$ & \multicolumn{2}{|l|}{$\eta_{T F}$} & $\eta_{T I}$ & $\eta_{P C R}$ \\
\hline 1 & \multicolumn{2}{|c|}{$0.0457(0.0389,0.0555)$} & $0.316(0.185,0.86)$ & $0.0697(0.0583,0.0824)$ & \multicolumn{2}{|l|}{-} & - & - \\
\hline 2 & \multicolumn{2}{|l|}{-} & - & - & \multicolumn{2}{|l|}{-} & - & - \\
\hline 3 & \multirow{2}{*}{\multicolumn{2}{|c|}{$0.0454(0.0366,0.057)$}} & $0.24(0.152,0.793)$ & $0.0708(0.058,0.0849)$ & \multicolumn{2}{|c|}{$0.0225(0.001,0.062)$} & $.33(0.145,0.522)$ & $0.182(0.098,0.328)$ \\
\hline 4 & & - & & - & \multicolumn{2}{|c|}{$0.0248(0.001,0.068)$} & - & - \\
\hline 5 & \multicolumn{2}{|c|}{$0.0385(0.0329,0.0443)$} & $0.596(0.368,0.875)$ & $0.0616(0.0534,0.0717)$ & - & & - & - \\
\hline 6 & \multicolumn{2}{|c|}{-} & & - & - & & - & - \\
\hline 7 & $0.0471(0.0373,0$ & $0.183(0.1$ & $32,0.609)$ & $0.0703(0.0583,0.0844)$ & - & & - & - \\
\hline 8 & - & - & & - & - & & - & - \\
\hline 9 & $0.984(0.98,0.98$ & $0.627(0.5$ & $75,0.887)$ & $0.963(0.957,0.966)$ & - & & - & - \\
\hline 10 & - & - & & - & - & & - & - \\
\hline
\end{tabular}

a: please see Additional file 1 for the interpretations of parameters; for the observation model with sensitivity and specificity (models 9 and 10 ), $\mu_{T F}$ is the sensitivity of TF, $\sigma_{T F}$ is the specificity of TF, $\mu_{T I}$ is the sensitivity of $\mathrm{Tl}, \sigma_{T \mathrm{~T}}$ is the specificity of $\mathrm{Tl}, \mu_{P C R}$ is the sensitivity of $\mathrm{PCR}$, and $\sigma_{P C R}$ is the specificity of PCR

: $95 \%$ Cl was obtained from MCMC with 16384 steps after a burn-in including 8192 steps 
Table 3 Estimated sensitivity and specificity

\begin{tabular}{llll}
\hline Test & $\begin{array}{l}\text { Sensitivity } \\
\text { Mean }(95 \% \mathrm{Cl})^{\mathrm{a}}\end{array}$ & $\begin{array}{l}\text { Specificity } \\
\text { Mean }(95 \% \mathrm{Cl})^{\mathrm{a}}\end{array}$ & $\begin{array}{l}\text { Delay in TF recovery (per six-month) } \\
\text { Mean }(95 \% \mathrm{Cl})^{\mathrm{a}}\end{array}$ \\
\hline Model 9 & $0.65(0.61,0.82)$ & $0.90(0.88,0.91)$ & $0.32(0.25,0.36)$ \\
$\mathrm{TF}$ & $0.58(0.51,0.78)$ & $0.98(0.98,0.99)$ & - \\
TI & $0.63(0.58,0.89)$ & $0.96(0.96,0.97)$ & - \\
PCR & & & $0.24(0.18,0.35)$ \\
Model 10 & $0.54(0.50,0.90)$ & $0.90(0.89,0.92)$ & \\
\hline TF &
\end{tabular}

a: $95 \% \mathrm{Cl}$ was obtained from MCMC with 16384 steps after a burn-in including 8192 steps

evaluated the sensitivity and specificity of diagnostic tests for ocular chlamydia infection including the clinical signs of TF and TI and PCR-based assay in the absence of a gold standard [3, 5, 25]. Recently, a regression model was used to forecast the TF prevalence after a number of years of mass drug administration given the ITI database [26]. A hidden Markov model was used to estimate the sensitivity and specificity of TF test based on the data collected from East and West Africa [5]. A latent class analysis was performed to estimate the sensitivity and specificity of TF test based on the data from randomly selected 40 villages in Ethiopia (the Trachoma Elimination Follow-up study) [3]. In [26], linear and logistic regression modeling was applied to a comprehensive database of trachoma prevalence to investigate the effect of MDA on baseline TF prevalence. In our previous study [27], we compared the forecasts of trachoma prevalence by expert opinion, statistical regression and transmission models using the data from 24 villages in Niger (PRET study). However, the delay in TF recovery was not included in $[3,5,26]$ and [27]; [3] and [26] did not use the process model; the forecasts made by [27] were only based on the observed PCR data.

Here, we forecasted trachoma in the short term of 6 months. Longer term models would be of more practical use to control programs and can be studied in the future. The process model used in this study did not include strain diversity [28], household-level risk factors [29], and infection from outside the population of children aged 05 years in each community [23]. Models could be further refined to reflect age-structured transmission. In this setting, the older children and adults were being treated as well, and other studies have shown consistently higher prevalence in small children than in other age groups (e.g. $[30,31])$. The use of results from 24 communities allowed us to separate the performance of several models-larger studies should be able to discriminate smaller differences. It should be noted that here, due to the available study data, we studied 0-5 year-old children, whereas the WHO criteria are defined in terms of 1-9 year-old children. Communities were geographically separated villages, although we cannot rule out transmission between villages.
In this study, we used a transmission model to forecast TF prevalence. The results demonstrated that using the delay in TF recovery in the model could improve the forecasts of TF prevalence. Because a recovery period may follow the clearance of infection and the follicles so characteristic of active trachoma may linger for weeks, in which case the clinical exam would falsely indicate the presence of Chlamydia trachomatis [32]. We did not find a significant difference between the forecasts of TF prevalence based on the observed TF data and based on the observed TF, TI and PCR data. Current WHO guidelines for starting MDA are based on the district prevalence of TF in children (aged 1-9 years), and the results in this study (based on children aged 0-5 years) suggest that future studies could consider use of the delay in TF recovery and TF data to assess forecasting at district level.

In many districts worldwide, the assessment of 2020 goals will likely include estimation of TF prevalence from surveys that had been done earlier than 2020 . These estimations could take into account the features found here to be useful in forecasting. It is also possible that as we near elimination, a laboratory indication of infection status supplants clinical signs such as TF-forecasting of a laboratory sign of infection might require a different analysis than that presented here.

\section{Additional files}

Additional file 1: Modeling methods. (PDF $336 \mathrm{~kb}$ )

Additional file 2: Observed TF, TI and PCR prevalence. (PDF $11 \mathrm{~kb}$ )

Additional file 3: Forecasts of TF prevalence versus observed TF prevalence. The forecast distributions of TF prevalence at 36-month in each of 24 communities from 10 models are shown by solid and dotted curves in different colors (as listed in the large legends), and their densities at 0 in each community are listed in the small legends. The observed TF prevalence in each community at 36-month is shown by the dashed grey bar. (TIFF $2817 \mathrm{~kb}$ )

Additional file 4: Forecasts of the hidden (true) prevalence. The forecast distributions of true prevalence at 36-month in each of 24 communities from 10 models are shown by solid and dotted curves in different colors (as listed in legends). (TIFF $1931 \mathrm{~kb}$ )

Competing interests

The authors declare that they have no competing interests. 


\section{Authors' contributions}

$T M L, T C P$ and FL conceived and designed the experiments. FL, TML, TCP, AA, $B K, B N, S K W, R L B$ and JDK performed the experiments. TML, TCP and FL analyzed the data. FL, TML and TCP wrote the manuscript. All authors read and approved the final manuscript

\section{Acknowledgments}

In addition to the study sponsors the Bill and Melinda Gates Foundation (grant number 48027) and MIDAS (grant number U01GM08778), the authors gratefully acknowledge funding of the NTD Modelling Consortium by the Bill and Melinda Gates Foundation in partnership with the Task Force for Global Health. The views, opinions, assumptions or any other information set out in this article are solely those of the authors. The authors thank the data and safety monitoring committee, including Douglas Jabs, MD, MBA (chair), Antoinette Darville, MD, Maureen Maguire, PhD, and Grace Saguti, MD, who were generous with their time and advice and met before and during the study. The authors thank all of our colleagues in Niger at Programme National de Santé Oculaire who collected samples and data. This trial is registered at ClinicalTrials.gov, number NCT00792922. We also thank the NTD support center and the Task Force for Global Health.

\section{Author details}

${ }^{1}$ F.I. Proctor Foundation, University of California San Francisco, 513 Parnassus, Medical Sciences 309A, San Francisco, CA 94143-0944, USA. ²Department of Ophthalmology, University of California San Francisco, San Francisco, CA, USA. ${ }^{3}$ Department of Epidemiology and Biostatistics, University of California San Francisco, San Francisco, CA, USA. ${ }^{4}$ Programme FSS/Université Abdou Moumouni de Niamey, Programme National de Santé Oculaire, Niamey, Niger. ${ }^{5}$ Dana Center for Preventive Ophthalmology, Wilmer Eye Institute, Johns Hopkins University, Baltimore, MD, USA. ${ }^{6}$ Clinical Research Unit, Department of Infectious and Tropical Diseases, London School of Hygiene and Tropical Medicine, London, UK.

Received: 31 August 2015 Accepted: 28 September 2015

Published online: 22 October 2015

\section{References}

1. Taylor HR, Burton MJ, Haddad D, West S, Wright H. Trachoma. Lancet. 2014;384(9960):2142-52.

2. Gambhir M, Pinsent A. Possible changes in the transmissibility of trachoma following MDA and transmission reduction: implications for the GET2020 goals. Parasites Vectors 2015, [In press].

3. See CW, Alemayehu W, Melese M, Zhou Z, Porco TC, Shiboski S, et al. How reliable are tests for trachoma? - a latent class approach. Invest Ophthalmol Visual Sci. 2011;52(9):6133-7.

4. Solomon AW, Peeling RW, Foster A, Mabey DC. Diagnosis and assessment of trachoma. Clin Microbiol Rev. 2004;17(4):982-1011.

5. Koukounari A, Moustaki I, Grassly NC, Blake IM, Basáñez M-G, Gambhir M, et al. Using a nonparametric multilevel latent Markov model to evaluate diagnostics for trachoma. Am J Epidemiol. 2013;177(9):913-22.

6. Bailey $R$, Arullendran P, Mabey D, Whittle H. Randomised controlled trial of single-dose azithromycin in treatment of trachoma. Lancet. 1993;342(8869):453-6.

7. Bailey R, Hampton T, Hayes L, Ward M, Whittle H, Mabey D. Polymerase chain reaction for the detection of ocular chlamydial infection in trachomaendemic communities. J Infect Dis. 1994;170(3):709-12.

8. Zucchini W, MacDonald IL. Hidden Markov models for time series : an introduction using R. Boca Raton: CRC Press; 2009.

9. Amza A, Kadri B, Nassirou B, Stoller NE, Yu SN, Zhou Z, et al. Community risk factors for ocular Chlamydia infection in Niger: pre-treatment results from a cluster-randomized trachoma trial. PLoS Negl Trop Dis. 2012;6(4):e1586.

10. Thylefors B, Dawson CR, Jones BR, West S, Taylor HR. A simple system for the assessment of trachoma and its complications. Bull World Health Organ. 1987:65(4):477.

11. Brauer $F$, van den Driessche $P$, Wu J. Mathematical Epidemiology. Berlin: Springer; 2008.

12. Ray KJ, Lietman TM, Porco TC, Keenan JD, Bailey RL, Solomon AW, et al. When can antibiotic treatments for trachoma be discontinued? Graduating communities in three african countries. PLoS Negl Trop Dis. 2009;3(6):e458.
13. Lietman TM, Gebre T, Ayele B, Ray KJ, Maher MC, See CW, et al. The epidemiological dynamics of infectious trachoma may facilitate elimination. Epidemics. 2011;3(2):119-24.

14. Ristic B, Arulampalam S, Gordon N. Beyond the Kalman filter. IEEE Aerosp Electron Syst Mag. 2004;19(7):37-8.

15. Team RC. R: A Language and Environment for Statistical Computing. 320th ed. Vienna: R Foundation for Statistical Computing; 2015.

16. Blake IM, Burton MJ, Solomon AW, West SK, Basáñez M-G, Gambhir M, et al. Targeting antibiotics to households for trachoma control. PLOS Negl Trop Dis. 2010;4(11):e862.

17. Grassly NC, Ward ME, Ferris S, Mabey DC, Bailey RL. The natural history of trachoma infection and disease in a gambian cohort with frequent followup. PLoS Negl Trop Dis. 2008;2(12):e341.

18. Michel C-EC, Solomon AW, Magbanua JP, Massae PA, Huang L, Mosha J, et al. Field evaluation of a rapid point-of-care assay for targeting antibiotic treatment for trachoma control: a comparative study. Lancet. 2006:367(9522):1585-90.

19. Lietman T, Porco T, Dawson C, Blower S. Global elimination of trachoma: how frequently should we administer mass chemotherapy? Nat Med. 1999;5(5):572-6.

20. Gambhir M, Basanez MG, Turner F, Kumaresan J, Grassly NC. Trachoma: transmission, infection, and control. Lancet Infect Dis. 2007;7(6):420-7.

21. Blake IM, Burton MJ, Bailey RL, Solomon AW, West S, Munoz B, et al. Estimating Household and Community Transmission of Ocular Chlamydia trachomatis. PLoS Negl Trop Dis. 2009;3(3):e401.

22. Liu F, Porco TC, Ray KJ, Bailey RL, Mkocha H, Munoz B, et al. Assessment of transmission in trachoma programs over time suggests no short-term loss of immunity. PLoS Negl Trop Dis. 2013;7(7):e2303.

23. Liu F, Porco TC, Mkocha HA, Munoz B, Ray KJ, Bailey RL, et al. The efficacy of oral azithromycin in clearing ocular chlamydia: mathematical modeling from a community-randomized trachoma trial. Epidemics. 2014;6:10-7.

24. Lietman TM, Gebre T, Abdou A, Alemayehu W, Emerson P, Blumberg S, et al. The distribution of the prevalence of ocular chlamydial infection in communities where trachoma is disappearing. Epidemics. 2015;11:85-91.

25. Keenan JD, See CW, Moncada J, Ayele B, Gebre T, Stoller NE, et al. Diagnostic characteristics of tests for ocular Chlamydia after mass azithromycin distributions. Invest Ophthalmol Vis Sci. 2012;53(1):235-40.

26. Jimenez V, Gelderblom HC, Mann FR, Emerson PM, Haddad D. Mass drug administration for trachoma: how long is not long enough? PLoS Negl Trop Dis. 2015;9(3):e0003610-0.

27. Liu F, Porco TC, Amza A, Kadri B, Nassirou B, West SK, et al. Short-term Forecasting of the Prevalence of Trachoma: Expert Opinion, Statistical Regression, versus Transmission Models. PLoS Negl Trop Dis. 2015;9(8):e0004000.

28. Bain DL, Lietman T, Rasmussen S, Kalman S, Fan J, Lammel C, et al. Chlamydial genovar distribution after communitywide antibiotic treatment. J Infect Dis. 2001;184(12):1581-8.

29. Last AR, Burr SE, Weiss HA, Harding-Esch EM, Cassama E, Nabicassa M, et al. Risk Factors for Active Trachoma and Ocular Chlamydia trachomatis Infection in Treatment-Naïve Trachoma-Hyperendemic Communities of the Bijagós Archipelago, Guinea Bissau. PLoS Negl Trop Dis. 2014;8(6):e2900.

30. Solomon AW, Holland MJ, Alexander ND, Massae PA, Aguirre A, NatividadSancho A, et al. Mass treatment with single-dose azithromycin for trachoma. N Engl J Med. 2004;351(19):1962-71.

31. Gambhir M, Singh BK, Michael E. Chapter One-The Allee Effect and Elimination of Neglected Tropical Diseases: A Mathematical Modelling Study. Adv Parasitol. 2015:87:1-31.

32. Miller K, Schmidt G, Melese M, Alemayehu W, Yi E, Cevallos V, et al. How reliable is the clinical exam in detecting ocular chlamydial infection? Ophthalmic Epidemiol. 2004;11(3):255-62. 